\title{
The OpenAIRE2020 FP7 Post-Grant Open Access Pilot: Implementing a European-wide funding initiative for Open Access publishing costs
}

\author{
Pablo De Castro \\ Stichting LIBER, The Hague, The Netherlands
}

\begin{abstract}
In the first half of 2015 the European Commission launched a new funding initiative to cover the Open Access publishing costs of publications arising from finished Seventh Framework Programme (FP7) projects. This article addresses the opportunities and challenges faced by this FP7 Post-Grant Open Access Pilot and discusses early project findings six months into this two-year initiative. This new and wide-scoped funding initiative arrives at a timely moment when a number of Gold Open Access funds are already in place at institutions in different European countries, which offers opportunities for promoting a gradual technical alignment of Article Processing Charges (APC) management practices. At the same time, there are rather large differences across Europe in the attitudes and researcher culture towards this emerging Gold Open Access business model which will need to be addressed within a swiftly evolving publishing landscape.
\end{abstract}

Keywords: Open Access, Gold Open Access, Open Access policies, Open Access publishing, Article Processing Charges, business models

\section{Introduction: The FP7 Post-Grant Open Access Pilot}

Following the "Guidelines on Open Access to Scientific Publications and Research Data in [the] Horizon 2020 [Research Framework Programme]" released on Dec. 11, 2013 by the European Commission [3], a new funding instrument was launched earlier this year in order to fund the Open Access publishing fees for publications arising from post-grant FP7 projects. This initiative, called the FP7 Post-Grant Open Access Pilot [2], is to be carried out within the OpenAIRE2020 project as an additional means of supporting the further spreading of Open Access across Europe. The pilot nature of the initiative involves an opportunity to carry out a thorough analysis on a swiftly evolving publishing landscape where Open Access journals are quickly becoming mainstream choices in many disciplines. This pilot character also points at the possibility of this funding mechanism being eventually extended if successful to support future post-grant Open Access publishing needs.

The OpenAIRE2020 FP7 Post-Grant OA Pilot, coordinated by LIBER (Association of European Research Libraries) in the Netherlands, manages a $€ 4 \mathrm{~m}$ Open Access publishing fund to be distributed among over 8000 eligible FP7 projects. The projects must have finished by the time they apply for funding (hence the post-grant character), but in order to ensure that the publications are still linked to the project activity, no more than two years should have passed since they reached their end-date. Eligible FP7 projects can be funded to publish up to three research articles, conference proceedings, 
monographs or edited volumes through the Gold Open Access route, i.e. by paying Article Processing Charges (APCs) to Open Access journals (or BPCs to Open Access book publishers) for their outputs to be openly available since their very publication. The initiative will run for a maximum of two years unless the funding is exhausted earlier.

The FP7 Post-Grant Open Access Pilot is being implemented by an international group of project partners which includes the University of Athens/Athena Research Centre in Greece, the University of Göttingen in Germany, the Jisc and the University College London (UCL) in the United Kingdom and SURF in the Netherlands. This partner network makes it possible to extend the project's reach and to identify and re-use previous working procedures where available. Furthermore, by bringing together institutions in different countries, opportunities arise to interact with similar funding initiatives taking place in these countries, thus contributing to the desired alignment of national and institutional funding strategies both at technical and at policy level.

Six months into this two-year project, it is already possible to come up with a number of achievements, challenges, early findings and mid-term objectives for this FP7 Post-Grant Open Access Pilot, which are summarized below. The description of the implementation process for this funding initiative focuses on three main areas of practice covering three different project worklines at different stages of completion: the workflows around APC management, the results collected so far and the APC-equivalent funding mechanism.

\section{Implementing the workflows around APC/BPC management}

One of the most pressing challenges for implementing an initiative to fund Open Access publishing fees is the sheer amount of administration required to deal with the many different aspects of the process, including information collection, funding request validation and invoicing [12]. This is arguably the main reason that makes institutions and libraries - especially small ones - unhappy about APC management. The eventual implementation of pre-payment agreements with publishers is due to significantly ease the administrative burden on all involved stakeholders, but in the meantime the FP7 Post-Grant Pilot has designed rather lightweight workflows on the institutions' side. By collecting, disseminating and re-using best institutional practices around its implementation and by taking most of the administrative work itself, this initiative is also an attempt to test a smooth approach to institutional APC management, where the emphasis lies in the dissemination effort.

The way the FP7 Post-Grant Open Access Pilot has tackled the APC management challenge is by designing and implementing a system [10] for automatically collecting and processing funding requests which reduces the amount of administration work on the user's side to the minimum possible. This system requests researchers or institutional staff applying for funding on their behalf to provide the minimum sufficient information for identifying the FP7 project, the publication and the desired amount of funding. This is done - regardless of the publication type - by relying on a number of embedded databases that will allow the system to autocomplete as many steps as possible in the information collection process. The resulting workflow involves a two-step validation process whereby funding requests are first conditionally approved if they meet the eligibility requirements stated in the policy guidelines [5]. Once an appropriate invoice is made available by the publisher, it may be uploaded in the system for final funding approval and processing by the pilot's accounting office.

The system workflows were designed by carefully analysing the publishing cycle and validating the early outcome against pre-existing best practices (see an early draft in Fig. 1). The University College 


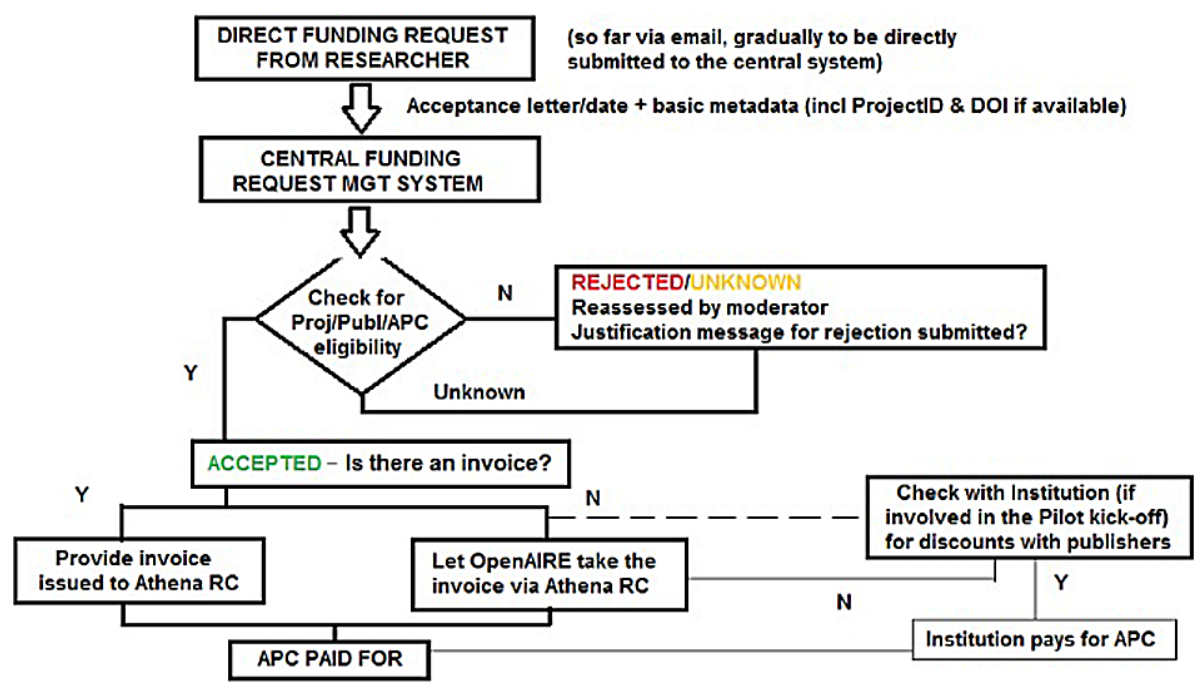

Fig. 1. Early FP7 Post-Grant Open Access Pilot workflow for funding request processing.

London (UCL), a partner in the initiative, was managing 2000 APCs a year at the time these workflows were put together [6]: this offered a unique opportunity to validate such workflow design.

Once the FP7 Post-Grant Open Access Pilot was officially kicked-off with the launching of the system for collecting and processing funding requests, the workflows went through a further and more comprehensive testing against the users' practices. A permanent fine-tuning loop was run in the first few months of operation to fix any identified flaws and to improve the system's user-friendliness towards researchers, librarians and research office staff at institutions.

Although originally designed to be as simple as possible, the FP7 Post-Grant Open Access Pilot policy guidelines include a number of clauses and requirements that are occasionally difficult to completely automate in the system. Some of these elements are the maximum of two years since the project's end-date, the fact that publications in hybrid journals are not eligible for funding or the maximum of three funded publications per eligible FP7 project and the subsequent need for the project coordinator to be notified about the incoming funding requests. Thanks to the system enhancement work done at the Athena Research Centre, all these features gradually found their way into the default automated workflow for funding request processing, which allowed the initiative to smoothly deal with the gradual increase in the number of processed requests as months went by.

\section{Some results six months into the project}

The FP7 Post-Grant Open Access Pilot is not an easy initiative to disseminate. The project coordinator, LIBER, is not a research-performing organization and has no researchers directly under its umbrella. The project aims to fund publications arising from completed FP7 projects that may or may not be produced. The area of expertise where the project takes place lies midway between the library - where the knowledge about specific Open Access aspects such as hybrid journals usually lies - and the research office or EU projects office, where the expertise on project management is, and will subsequently require an effective collaboration between both of them for the initiative to be successfully disseminated at institutional level. 
The most difficult challenge lies however in the need to raise awareness of the funding initiative directly among researchers, since they are the ones who will need to bear it in mind at manuscript acceptance or even at manuscript submission time. This may be slightly easier in countries where previous exercises in Gold Open Access funding have taken place or are taking place, as institutional libraries and research offices will only need to add an additional available funding source to the information they're already disseminating to their affiliated researchers. However, this FP7 Post-Grant OA Pilot aims to provide funding across many different countries, most of which have no pre-existing Gold Open Access funding workflows in place. Furthermore, the project aims to distribute the funding in a reasonably balanced way across such countries.

Together with the fact that the Pilot was launched nearly in summertime, this lack of pre-existing workflows was the main reason why the partners involved in the project suspected it was likely to have a slow take-off [14]. Logic said that the dissemination strategies would need to start working before any significant growth in the number of approved funding requests could be expected to take place. In fact this is exactly the way things went until an early October dissemination action changed the course of the initiative: a letter was sent out from the European Commission to every FP7 project coordinator letting them know that a new funding initiative had been launched to cover the Open Access publishing fees for their post-grant publications. This meant a nearly instant upsurge in the number of collected funding requests. The direct communication to researchers was also very well timed, as it was sent once the system for collecting and processing funding requests had been sufficiently fine-tuned to cope with the spike in the number of submissions.

As a result of the combined dissemination strategy, which also included many project presentations at events in different countries by the coordination and OpenAIRE's NOADs, the first 100 granted funding request milestone was eventually reached on Nov. 12, i.e. a little over 5 months after the actual Pilot kick-off [1]. Figure 2 shows the gradual growth in the number of approved funding requests.

While the sample is not large enough yet to be significant for statistical purposes, the distribution of approved funding requests by country in Table 1 below already shows countries where previous Gold

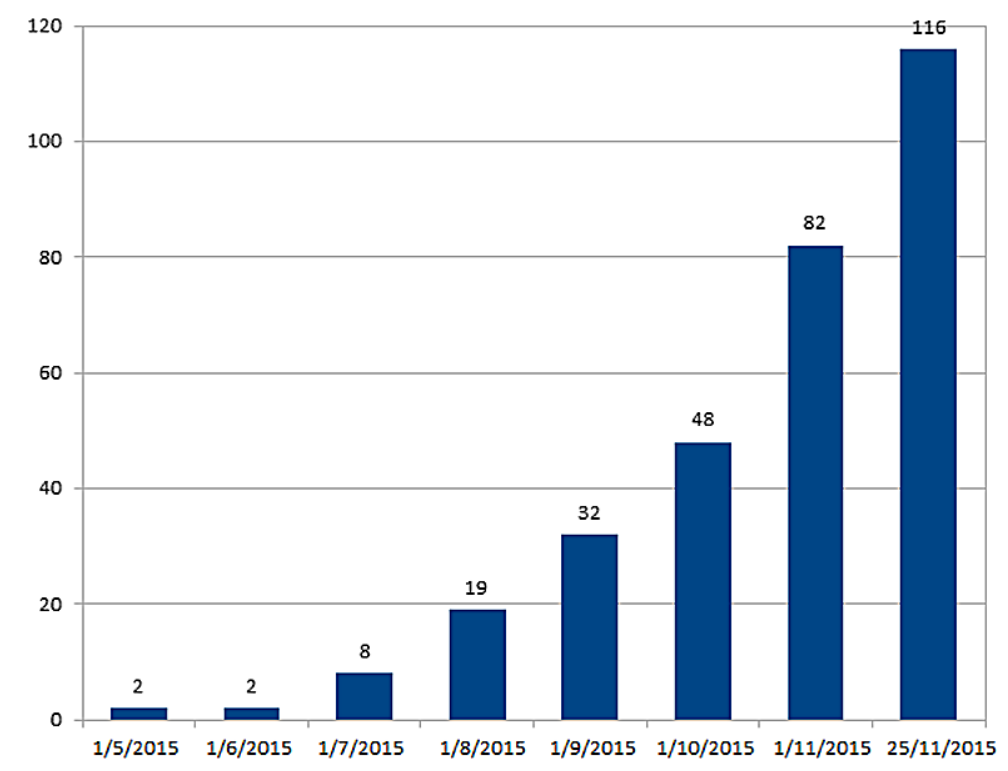

Fig. 2. Monthly evolution in the number of funding requests granted by the EC FP7 Post-Grant Open Access Pilot. 
Table 1

Distribution of approved funding requests by [requestor's] country

\begin{tabular}{lc}
\hline Country & Approved funding requests (as of Nov. 25, 2015) \\
\hline ES & $29(+17)$ \\
UK & $11(+8)$ \\
IT & $8(+4)$ \\
CH & $8(+3)$ \\
DE & $5(+4)$ \\
NL & $4(+4)$ \\
FR & $4(+3)$ \\
GR & $3(+3)$ \\
BE & $3(+2)$ \\
SE & $3(+2)$ \\
NO & $3(+2)$ \\
PT & $3(+1)$ \\
DK & $2(+2)$ \\
IL & $2(+2)$ \\
TR & $2(0)$ \\
HU & $1(+1)$ \\
CZ & $1(+1)$ \\
IE & $1(+1)$ \\
BF & $1(+1)$ \\
PL & $1(0)$ \\
AT & $1(0)$ \\
ZA & $116(+64)$ \\
Total & \\
Notes: Figures in brackets show the growth since the last report was \\
released with data as of Sep. 30.
\end{tabular}

Open Access funding initiatives are in place do not necessarily have a position of privilege for benefiting from this funding opportunity. This is mainly because researchers in these countries, even those funded by eligible FP7 projects, will often find it easier to collect the funding for their APCs from these preexisting national or institutional Gold Open Access funds they already know about than from a new one whose policy they need to learn to navigate.

Another early finding from the analysis of the provenance of the funding requests that have been granted so far is the fact that the share of approved requests awarded to research centres and university hospitals is far larger (nearly $40 \%$ of the total number of approved requests at the moment) than foreseeable when comparing their number to the number of universities involved in FP7 projects. This may hint at the fact that workflows for handling funding requests for APCs are getting more effectively implemented at research centres, where libraries have a full focus on research support, than at universities, whose libraries have numerous additional tasks linked to supporting teaching and students.

Data is also being collected and analyzed for the APC fees paid so far, a summary of which is being released as part of the bimonthly reporting for this initiative. Figures from the last report released early Oct. with data as of Sep. 30 [7] show an average APC of $€ 1352$ for the 52 approved funding requests available at the time. This is a slightly higher average value than the $€ 1230.4$ one collected for the German Research Foundation's co-funded Gold Open Access Fund at German research institutions [11], whose no-hybrid policy is fully aligned with the FP7 Post-Grant Open Access Pilot's. An informal 
coordination initiative is however under way across Europe around APC management [13] to increase transparency on the APC fees paid in different countries and to make sure that the APC payment datasets collected by the different funding initiatives have a common structure so that they can be exchanged and compared.

Moreover, the FP7 Post-Grant Open Access Pilot is making publicly available the list of fully Open Access journals which have collected funding from the project [8]. This information was requested by institutions, who are increasingly aiming to provide their researchers more detailed information on what fully Open Access titles they can choose to submit their manuscripts to in order to be eligible for funding. The list of journal titles made available by the FP7 Post-Grant Open Access Pilot will be kept updated and will provide an insight into the growing range of options that researchers have at their disposal.

\section{The APC-equivalent funding mechanism}

Besides funding APCs and BPCs for publications arising from eligible FP7 projects whose authors choose to submit to publishers running author-pays business models, this FP7 Post-Grant Open Access Pilot also aims to implement an alternative funding mechanism by which funding will also be offered to APC-free Open Access journals: the APC-equivalent funding mechanism [9].

This is because being a Gold Open Access funding initiative, this FP7 Post-Grant Open Access Pilot is acutely aware of the fact that over two thirds of the journals listed in the Directory of Open Access Journals (DOAJ) do not charge APCs to their authors, and it will therefore try to seek a balance which also reinforces the APC-free Open Access journal infrastructure.

Questions to be considered in setting up such a mechanism include the need to have similar workflows to those applied to deal with funding requests for APC payments, plus a clear set of criteria to define funding eligibility for journals.

Instead of funding Article Processing Charges, which is a business model that the FP7 Post-Grant Open Access Pilot does not wish to promote among APC-free Open Access journals whose editors have regularly declared they find unsustainable, this APC-equivalent funding mechanism will fund technical improvements in the publishing workflows for such APC-free Open Access journals published in the 33 countries that participate in the OpenAIRE project. Fundable technical enhancements for APC-free Open Access journals will include among others aspects like collecting the funding information for their published articles (where available) in a specific metadata element (also retrospectively), producing XML versions of the published articles, having their contents indexed at article level by the DOAJ and OpenAIRE or implementing ORCID.

Same as it's being done for APC funding, this alternative funding mechanism will allocate funding to APC-free Open Access journals based on the number of articles they publish that arise from eligible post-grant FP7 projects, and this is where one of the most inspiring challenges lies for these journals. The FP7 Post-Grant Open Access Pilot has been discussing the way to implement this initiative with a number of APC-free Open Access journals and journal platforms during the last few months such as the Open Library of Humanities in the UK, EKT ePublishing in Greece, Revistas CSIC in Spain, UOpen in The Netherlands or the Hrčak Portal in Croatia, and there is a widespread consensus on the fact that it's not frequent for these APC-free Open Access journals to collect submissions from FP7 projects. Some mechanism will need to be identified and implemented for these journals - most of which belong to disciplines in the Social Sciences and Humanities - to become more attractive to researchers and start receiving submissions that will allow them to become eligible for funding. The strengthening of 
their technical publishing workflows will certainly be part of this effort to be increasingly perceived as interesting options to submit manuscripts to, but the objective of this alternative funding mechanism is also to make them more proactive with regard to their potential authors.

A workshop will be hosted on Dec. 11 by the FP7 Post-Grant Open Access Pilot at the Royal Library of The Netherlands in The Hague where the different aspects of the funding will be discussed with a number of attending APC-free Open Access journal editors and platform managers [4]. Funders will also be attending the workshop who have expressed an interest in this APC-equivalent funding mechanism, which the 2-year FP7 Post-Grant Open Access Pilot would be keen to see followed-up by nationallevel initiatives as a means for the funding opportunities for these journals to become more sustained in the mid-term. By bringing together different stakeholders involved in the ongoing discussion around funding models for non-APC-based Open Access journals, this APC-equivalent funding initiative aims to explore yet another opportunity to promote technical and policy alignment.

The work leading to the definition and implementation of this APC-equivalent funding mechanism will be particularly useful within the remit this FP7 Post-Grant Open Access Pilot has to gather evidence to create a roadmap for fostering a sustainable and competitive market for Open Access publishing.

\section{References}

[1] 100th granted funding request from the EC FP7 Post-Grant Open Access Pilot, OpenAIRE Newsletter, available at: https://www.openaire.eu/100th-granted-funding-request-from-the-ec-fp7-post-grant-open-access-pilot (retrieved Nov. 16, 2015).

[2] EC FP7 Post-Grant Open Access Pilot homepage, available at: https://www.openaire.eu/postgrantoapilot.

[3] European Commission, Guidelines on Open Access to scientific publications and research data in Horizon 2020 (Dec. 2013), available at: https://www.openaire.eu/guidelines-on-open-access-to-scientific-publications-and-research-data-inhorizon-2020 (retrieved Feb. 28, 2015). An updated version of the Guidelines (v2.0) was released on Oct. 30, 2015, available at: http://ec.europa.eu/research/participants/data/ref/h2020/grants_manual/hi/oa_pilot/h2020-hi-oa-pilot-guide_en. pdf.

[4] FP7 Post-Grant OA Pilot Workshop on the APC-equivalent funding mechanism, available at: https://www.eventbrite. co.uk/e/fp7-post-grant-oa-pilot-workshop-on-the-apc-equivalent-funding-mechanism-tickets-19740928649 (retrieved Nov. 26, 2015).

[5] FP7 Post-Grant Open Access Pilot policy guidelines, available at: https://www.openaire.eu/postgrantoapilot.

[6] S. Lawson and C. Sharp, UCL APCs 2014, figshare, available at: http://dx.doi.org/10.6084/m9.figshare.1305066 (retrieved Nov. 25, 2015).

[7] OpenAIRE blog, Second FP7 Post-Grant Open Access Pilot progress report, available at: https://blogs.openaire.eu/?p=530 (retrieved Nov. 25, 2015).

[8] OpenAIRE blog, Funded journal titles by the EC FP7 Post-Grant Open Access Pilot, available at: https://blogs.openaire. $\mathrm{eu} / \mathrm{p}=586$ (retrieved Nov. 25, 2015).

[9] OpenAIRE blog, "Not Just APCs": the FP7 Post-Grant OA Pilot's alternative APC-equivalent funding mechanism, available at: https://blogs.openaire.eu/?p=453 (retrieved Nov. 25, 2015).

[10] OpenAIRE system for collecting funding requests, available at: https://postgrantoapilot.openaire.eu/.

[11] D. Pieper, Open APC data in Germany - A contribution to Open Access monitoring, presentation delivered at the LIBER Annual, available at: http://www.slideshare.net/Dirk_Pieper/openapc-liber2015 (retrieved Nov. 25, 2015).

[12] S. Pinfield, J. Salter and P.A. Bath, The 'total cost of publication' in a hybrid open-access environment: Institutional approaches to funding journal article-processing charges in combination with subscriptions, Journal of the Association for Information Science and Technology (2015), to appear, available at: http://eprints.whiterose.ac.uk/83525/ (retrieved Nov. 22, 2015).

[13] See for instance the coordination activity carried out by the Knowledge Exchange Open Access Expert Group, available at: http://www.knowledge-exchange.info/projects/project/open-access.

[14] The strategy to follow in order to implement the FP7 Post-Grant Open Access Pilot across a fragmented geographic landscape was addressed in the Apr. 9, 2015 workshop held in The Hague with a number of institutions involved in the kick-off stage for the initiative, available at: https://www.openaire.eu/first-workshop-on-post-grant-gold-oa-pilot (retrieved Nov. 25, 2015). 